\title{
Coating Distribution in a Commercial SCR Filter
}

\author{
Mark L. Stewart ${ }^{1}$ (D) Carl Justin Kamp ${ }^{2,3} \cdot$ Feng Gao $^{1} \cdot$ Yilin Wang ${ }^{1} \cdot$ Mark H. Engelhard $^{4}$ \\ Received: 9 February 2018 / Revised: 1 August 2018 / Accepted: 14 August 2018 / Published online: 31 August 2018 \\ (C) The Author(s) 2018
}

\begin{abstract}
A commercial SCR filter, deployed in the USA in 2015, was sectioned and examined using techniques including mercury porosimetry, electron microscopy, and micro-X-ray computed tomography. The catalyst washcoat was found to be consistent with $\mathrm{Cu} / \mathrm{SSZ}-13$, possibly including some zirconia and alumina. Three distinct regions were observed with respect to catalyst loading and location. A region at the inlet end of the filter, comprising 15 to $21 \%$ of the total effective filter length, was relatively lightly coated. Most of the catalyst present in this region was observed inside the porous filter walls, and the catalyst concentration was generally greater near the upstream filter wall surfaces. Moving axially down the monolith toward the outlet, a second region comprising 14 to $20 \%$ of the total effective filter length was more heavily coated, with catalyst present throughout the thickness of the porous filter walls, as well as coatings on both the upstream and downstream filter wall surfaces. The final region at the outlet end of the monolith, which accounted for 65 to $70 \%$ of the filter length, had an intermediate catalyst loading. Most of the catalyst here was again observed inside the porous filter wall. Concentrations in this region were higher near the downstream filter wall surfaces. Detailed models of multi-functional aftertreatment devices, such as the one examined here, have included representations of catalyst distribution within the filter bricks and indicate that catalyst distribution may have an impact on flow distribution, soot loading patterns, local concentrations, and ultimately conversion efficiency. Previous work has also shown that catalyst distribution across the thickness of an exhaust filter wall can have significant impacts on backpressure during soot loading.
\end{abstract}

Keywords Diesel particulate filter $\cdot$ Selective catalytic reduction $\cdot$ X-ray computed tomography $\cdot$ Catalyzed exhaust filter $\cdot$ Catalyst coating

\section{Background}

Diesel particulate filters (DPFs) now play an important role in reducing harmful emissions from on-road vehicles. Deployment of exhaust particulate filters on gasoline vehicles has also begun, and will become more widespread as the result of tightening particle number standards in Europe and Asia, as well as new high-efficiency engine technologies [1]. As exhaust aftertreatment systems become more complex,

Mark L. Stewart

mark.stewart@pnnl.gov

1 Pacific Northwest National Laboratory, 902 Battelle Blvd, Richland, WA 99352, USA

2 Kymanetics Inc., Boston, MA 02152, USA

3 Massachusetts Institute of Technology, Cambridge, MA 02139, USA

4 Environmental Molecular Sciences Laboratory, Pacific Northwest National Laboratory, 3335 Innovation Blvd, Richland, WA 99352, USA combining multiple functionalities into fewer units offers advantages in minimizing cost and vehicle weight, while saving limited space. In some cases, cold-start performance can also be improved by moving catalysts closer to the engine [2].

Catalyst coatings with oxidation functionality were applied successfully to DPFs early in their commercialization to assist in filter regeneration, to reduce hydrocarbon and carbon monoxide emissions, and to promote subsequent unit operations downstream, for example, by increasing the ratio of $\mathrm{NO}_{2}$ to NO. Adding other functionalities, such as three-way catalysts (TWC) [3] or ammonia selective catalytic reduction (SCR) [4], is more challenging, in part because of the volume of catalyst necessary to achieve desired conversions, as well as competition with soot oxidation reactions. Unless applied carefully, catalyst coatings in exhaust filters can dramatically increase backpressure, especially as the filters accumulate soot $[4,5]$. Nevertheless, considerable progress has been made, and SCR filters are now available commercially $[6,7]$. The distribution of catalyst within multi-functional filters (axial, radial, and across the thickness of filter walls) likely impacts backpressure, filtration performance, and conversion of gaseous pollutants 
under various operating conditions [8]. Therefore, techniques for characterization of catalyst distribution could be valuable tools for researchers, filter manufacturers, catalyst coaters, and vehicle manufacturers, as they work together to optimize aftertreatment systems. As aftertreatment system characteristics, including catalyst distribution within individual components, continue to increase in complexity, establishing standard characterization methods also becomes important. This work describes characterization of catalyst distribution throughout the volume of one commercially produced SCR-filter brick using a combination of approaches including physical sectioning, optical examination, mercury porosimetry, microscopy, and X-ray computed tomography (CT).

\section{Approach}

\subsection{Device Examined}

The SCR catalyst-coated filter examined here was deployed with the Volkswagen 2.0-L TDI Clean Diesel EA288 engine. An aftertreatment sub-assembly (part number 04L131606M) intended for a 2015 Passat was disassembled and the filter brick removed. The entire sub-assembly is shown in Fig. 1. The filter brick is contained in the larger housing on the right. The filter brick (Fig. 2) was approximately $16.5 \mathrm{~cm}$ in diameter by $14 \mathrm{~cm}$ long. The filter was segmented silicon carbide (SiC) with approximately 300 cells per inch. The cells were asymmetric, with larger inlet channels and smaller outlet channels. The brick consisted of 17 segments having three cross-sectional shapes: four square segments in the center, four triangular segments in the corners, and eight partial segments on the edges.

It is the authors' understanding that an additional flowthrough SCR was included downstream in an under-floor position on these vehicles, in which case the SCR-coated filter only contained a fraction of the SCR catalyst in the entire aftertreatment system.

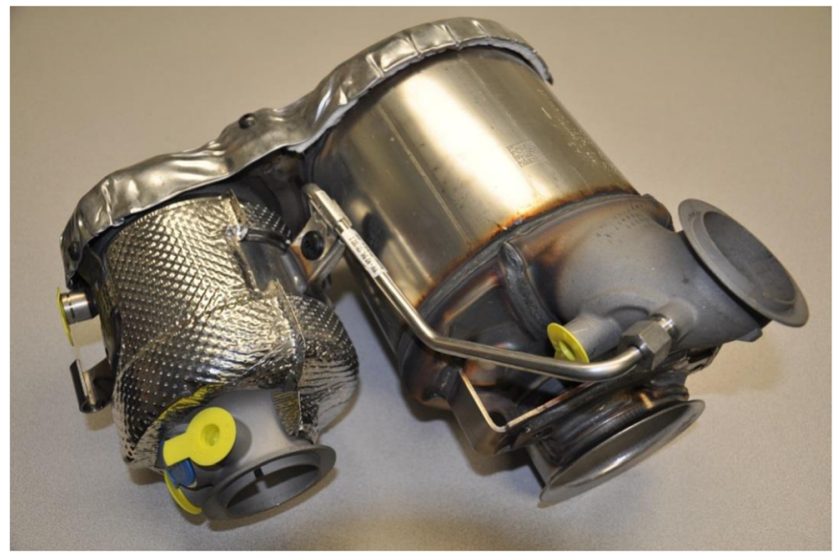

Fig. 1 Aftertreatment sub-assembly

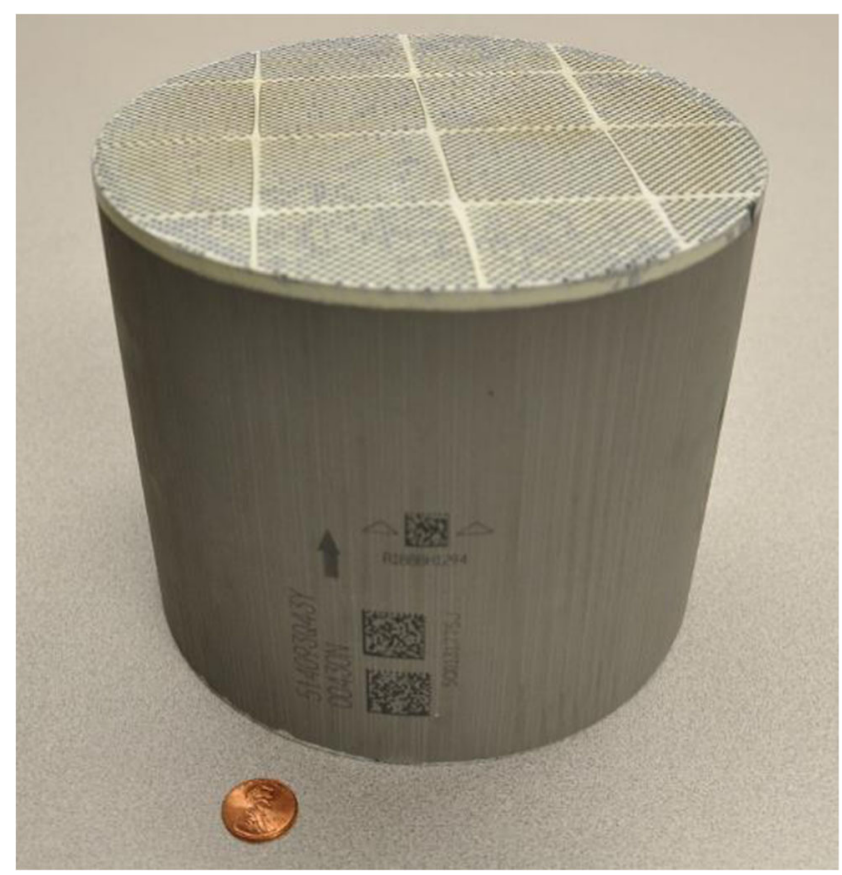

Fig. 2 Coated filter brick

Figure 3 shows a simplified schematic with nomenclature used throughout the paper to describe various locations within the part examined. The terms "inlet" and "outlet" are used to refer to both the respective axial ends of the filter monolith, and to the inlet channels (plugged at the outlet end) and outlet channels (plugged at the inlet end). Exhaust is forced through the porous filter walls from the upstream wall surface (in the interior of the inlet channels) to the downstream wall surface (in the interior of the outlet channels). Axial regions 1, 2, and 3 will be described in the "Results" section.

\subsection{Equipment and Methods}

Mercury porosimetry was performed with a Micromeritics Autopore 9600. The samples analyzed were small sections $(0.37-0.52 \mathrm{~g})$ taken from three different axial locations along one of the square central segments. The pore size distributions

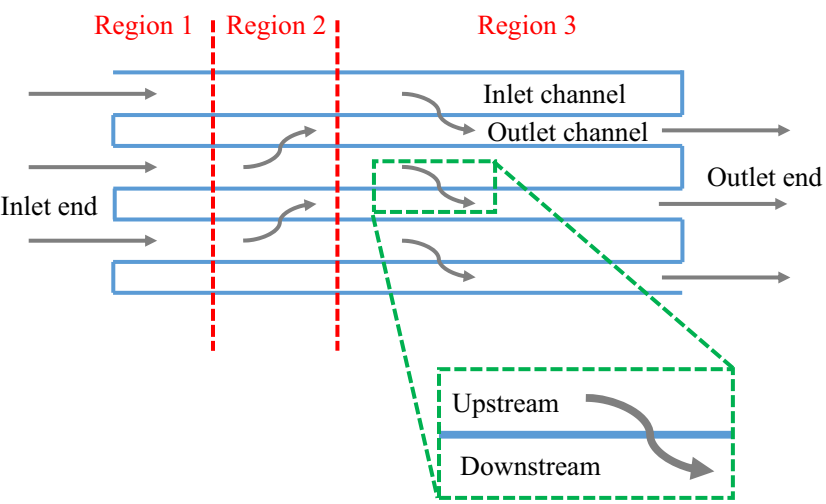

Fig. 3 Sketch showing position nomenclature used 
were modified slightly from the raw data. Pores larger than $50 \mu \mathrm{m}$ were assumed to be associated with surface roughness, not porosity internal to the filter walls, and are therefore not reflected in the pore size distributions or total porosity values shown.

X-ray data was collected using two different systems:

An entire edge segment and approximately half of a second edge segment were successively sectioned and imaged using an XTEK 225 micro-CT system at the Harvard University Center for Nanoscale Systems. This system utilizes a $16^{\prime \prime} \times$ $16 "$ PerkinElmer detector screen with multiple X-ray sources, including a bulk tungsten reflection source capable of approximately $1-\mu \mathrm{m}$ voxel size and a tungsten film transmission source capable of approximately $600-\mathrm{nm}$ voxel size. The XTEK 225 system has a large enclosure volume capable of scanning full-size aftertreatment components $(\sim 25 \mathrm{~L})$ down to single and sub-channel filter extractions. Multiple specific techniques are required to attain reproducible $1-\mu \mathrm{m}$ and submicron resolution with adequate contrast, including X-ray filtration, a custom-designed sample stage, and optimized X-ray conditions (voltage, current, sample rotation speed, exposure time, source focus), as discussed in [9]. Data was collected from the SCR-filter specimens at resolutions ranging from approximately $50 \mu \mathrm{m}$ per voxel down to $1 \mu \mathrm{m}$ per voxel.

Three small two-by-two channel samples were also removed from an edge segment and examined using a Bruker SkyScan 1272 high-resolution micro-CT system with a Hamamatsu L11871 X-ray source and a Ximea xiRay16 camera. The camera to source distance was $273 \mathrm{~mm}$, and the object to source distance was $61.56 \mathrm{~mm}$. The resulting image pixel size was $1.67 \mu \mathrm{m}$. The samples submitted for analysis were roughly $2-$ $2.5 \mathrm{~cm}$ long. Lengths actually scanned were roughly $13-14 \mathrm{~mm}$.

A number of electron micrographs were also taken of filter wall surfaces at various locations using a Phillips/FEI XL30 FEG environmental scanning electron microscope (ESEM) in the backscattered electron detector configuration.

A coating powder sample was scraped from the filter walls in a heavily coated region and examined by X-ray diffraction (XRD) and X-ray photoelectron spectroscopy (XPS). Another sample of crushed filter wall and the catalyst it contained was also examined by XRD. XRD measurements were performed on a Philips PW3040/00 X'Pert powder X-ray diffractometer with $\mathrm{Cu}-\mathrm{K} \alpha$ radiation $(\lambda=1.5406 \AA)$. Data were collected with $2 \theta$ ranging from 5 to $50^{\circ}$ using a step size of $0.02^{\circ}$. XPS was performed using a Physical Electronics Quantera Scanning X-ray Microprobe. This system uses a focused monochromatic Al K $\alpha$ X-ray $(1486.7 \mathrm{eV})$ source for excitation and a spherical section analyzer. The instrument has a 32 element multi-channel detection system. The $80-\mathrm{W}$ X-ray beam focused to $100-\mu \mathrm{m}$ diameter was rastered over a $1.1 \times 0.1-\mathrm{mm}$ rectangle on the sample. The X-ray beam was incident normal to the sample, and the photoelectron detector was at $45^{\circ}$ off-normal. High-energy resolution spectra were collected using a pass-energy of $69.0 \mathrm{eV}$ with a step size of $0.125 \mathrm{eV}$. Note that the samples experienced variable degrees of charging. Low-energy electrons at $\sim 1 \mathrm{eV}, 20 \mu \mathrm{A}$, and lowenergy $\mathrm{Ar}^{+}$ions were used to minimize this charging.

\subsection{Segmentation and Three-Dimensional Reconstruction}

Raw data obtained with the XTEK 225 system was reconstructed with the X-ray 3D Pro software from Nikon. Reconstruction for scanned samples in this study utilized multiple algorithms including noise reduction and beam hardening correction to optimize three-dimensional data clarity and contrast. Data segmentation, i.e., specifically identifying the density phase boundaries for the phases of interest, was done via the VGStudio Max software and the Avizo software by FEI. Since there were essentially three phases of interest, substrate, washcoat, and air, segmentation was fairly straightforward and was done via thresholding. (Note that segmentation of reconstructed three-dimensional X-ray CT data of wallflow aftertreatment components, such as the DPF, gasoline particulate filter, or SCR filter, becomes increasingly difficult when soot and/or lubricant-derived ash is present.) The relative densities of the three phases present in these samples were different enough that thresholding was simple and could be validated, e.g., with porosity values measured by other means. The reconstructed raw data was despeckled (to remove outlier voxels) before segmentation to decrease the noise.

Two-dimensional images of the three two-by-two channel samples from the SkyScan CT system were combined into three-dimensional datasets using a custom program written in MATLAB. Images were read, manipulated, and analyzed using the MATLAB Image Processing Toolbox. A total of 1600 frames were used for each sample, corresponding to an axial length of approximately $2.67 \mathrm{~mm}$. The CT data was coarsened two levels, resulting in a spatial resolution of $6.68 \mu \mathrm{m}$, in order to reduce noise and computational time for the analysis. Two masking arrays were chosen to define approximate three-dimensional volumes in the images: the first volume included both the filter substrate and catalyst coating, with any internal macro-porosity, and the second excluded thick surface coatings on the substrate wall, but included catalyst inside the substrate wall, again with any internal porosity. The masking arrays allowed the large void volumes surrounding the samples and in the honeycomb channels to be excluded, defining volumes that would correspond to those reflected in the mercury porosimetry data. The masking arrays were generated using the MATLAB bwareaopen and imclose functions, a technique commonly used to help define discrete objects in machine vision applications. A disk structural element with a diameter of 14 pixels was used in imclose. Approximate grayscale thresholds were manually iterated at this stage, in order to generate reasonable masking volumes. 
Once chosen, the same approximate thresholds were used to create masking arrays for all three of the two-by-two channel samples. Besides defining volumes within the threedimensional datasets for comparison to mercury porosimetry data, the masking arrays also allow estimates of how much catalyst is contained within the porous filter walls rather than coated on the filter wall surfaces.

Segmentation between the void, substrate, and catalyst coating phases was then accomplished by choosing two (more precise) grayscale thresholds for each dataset. Ideally, mercury porosimetry and X-ray CT data would be available to observe the porosity and pore morphology for the bare, uncoated substrate. Since that was not the case here, a porosity of $62 \%$ was assumed for the substrate. This value seemed consistent with the mercury porosimetry data for the coated filter and corresponds to advertised porosities for advanced, high-porosity substrates offered commercially for SCR-filter applications $[6,10]$. After applying a Wiener filter function to reduce high-frequency noise, a grayscale histogram was generated for the volume defined by the wall masking array. A grayscale threshold was then chosen that would yield the assumed substrate porosity in this volume. A similar process was then repeated in the volume approximating the substrate plus surface coating. This time the second threshold, defining the boundary between the catalyst coating and void, was chosen to yield the target resolved porosity in the axial filter location corresponding to the specific CT dataset.

The concept of "resolved porosity" was used to relate the porosity measured by mercury porosimetry to that visible in the CT images. With a spatial resolution of $6.67 \mu \mathrm{m}$ in the coarsened dataset, it is obvious that a portion of the porosity measured by mercury porosimetry would not be reflected in the CT data. In addition, some of the internal pore bodies that could be resolved in the CT images might be "screened" by smaller pore throats, or by regions filled with catalyst. On the other hand, some of this internal pore volume could be accessible to intruding mercury if there were some plastic deformation of catalyst deposits at high pressures. In the study described here, it was assumed that voxels associated with the catalyst coating had an unresolved porosity of $25 \%$. This allowed the total porosities measured by mercury porosimetry to be converted to the resolved porosity values used in the image segmentation. The actual total porosity within a volume occupied by catalyst would depend upon factors such as the size distribution and shape of the catalyst particles. Dudák et al. [11] gave a porosity value of $32 \%$ for a zeolite layer and $17 \%$ for a compact alumina layer.

\section{Results}

\subsection{Sectioning and Appearance}

A number of features were discernable to the naked eye as the coated filter brick was progressively sectioned. Figure 4 shows the interior of an edge segment. The section cuts across multiple channels, exposing both upstream filter wall surfaces in inlet channels and downstream filter wall surfaces in outlet channels. The inlet end of the filter is on the left, and the outlet end on the right. The most striking features are pronounced, light-colored regions close to the inlet end, indicative of thicker catalyst coatings. These regions with thick coatings were observed in approximately the same location in all segments examined, although their shapes and sizes varied. In most cases, the light coloration and associated thick surface coatings extended across the inlet and outlet channels.

At the inlet end of the filter (to the left of the very lightcolored, heavily coated regions in Fig. 4), the upstream filter wall surfaces in the inlet channels were a noticeably lighter color than those in the outlet channels. In the final axial region following the very light-colored, heavily coated region, the opposite pattern was observed, with somewhat lighter

Fig. 4 Sectioned filter segment

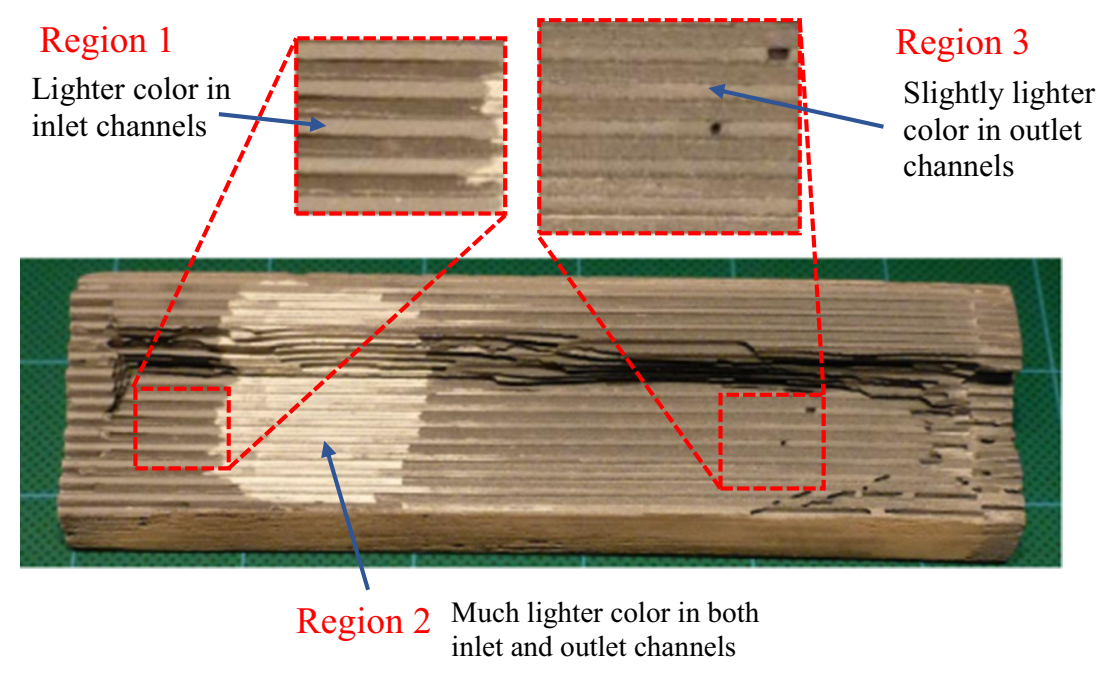




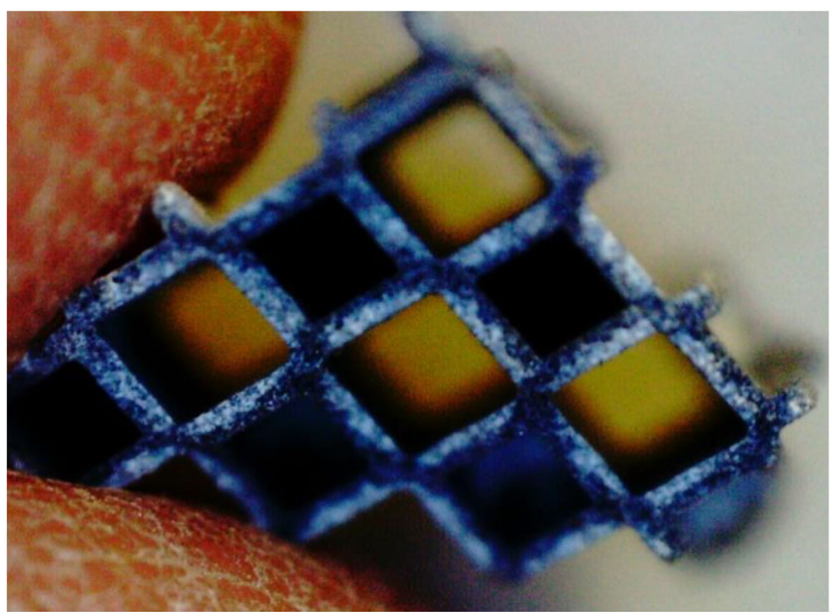

Fig. 5 Filter wall cross sections near inlet

downstream wall surfaces in the outlet channels, although here the difference was more subtle. The three distinct axial regions will be referred to as region 1 (inlet end), region 2 (light colored, heavily coated region near inlet), and region 3 (outlet end).

In some locations, it was possible to discern a gradient in color across the thickness of broken filter walls. Figure 5 shows the cross section of a small sample extraction taken from the inlet end of the brick. Lighter color in the filter walls can be seen radiating outward from the larger inlet channels.

\subsection{Microscopy}

A typical ESEM image of the upstream filter wall surface in region 3 is shown in Fig. 6. The underlying structure of sintered $\mathrm{SiC}$ grains is visible, as are a number of pore mouths at the filter wall surface. The smaller particles that make up the catalyst washcoat are visible as a thin coating on some of the $\mathrm{SiC}$ grains. The

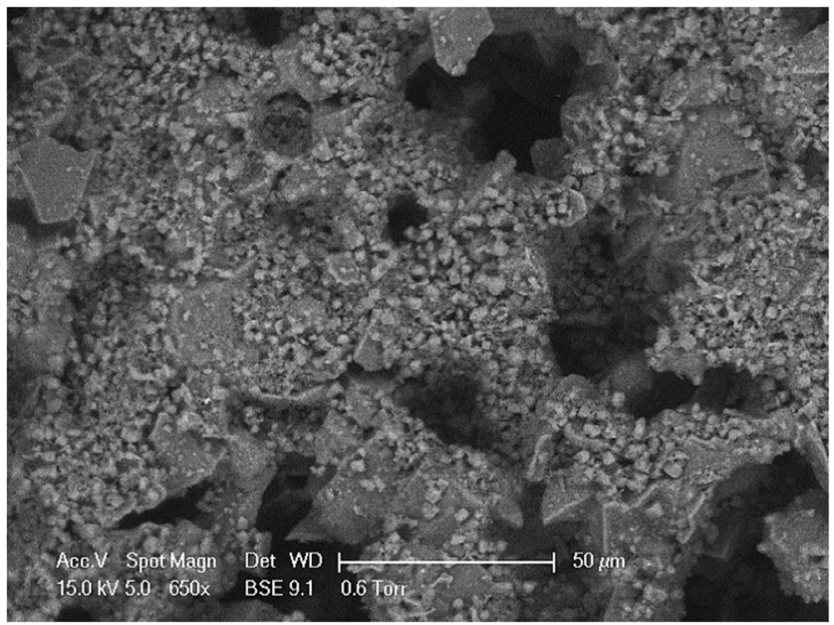

Fig. 6 Electron micrograph of upstream filter wall surface near outlet end of filter

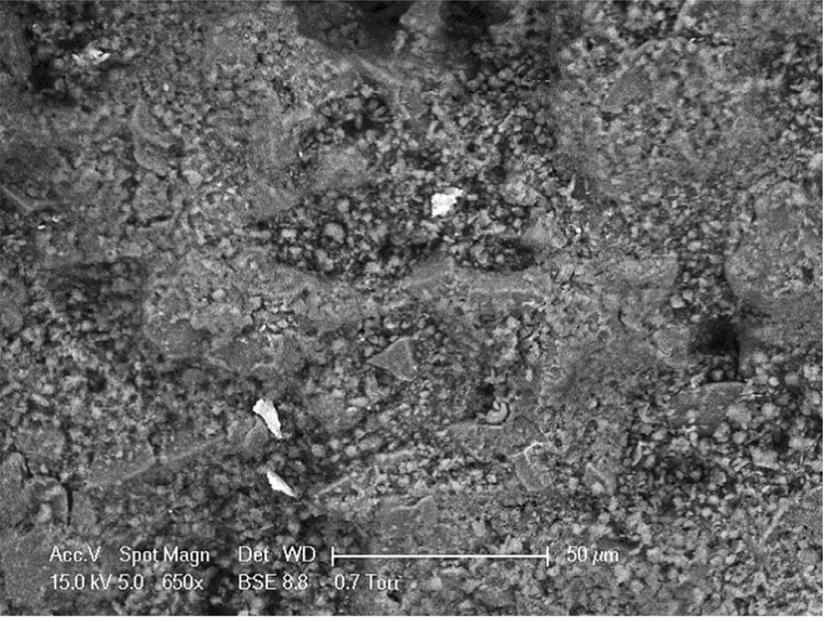

Fig. 7 Electron micrograph of downstream filter wall surface near outlet end of filter

downstream side of the filter wall at a similar axial location is shown in Fig. 7. Here, the underlying structure of the filter substrate is still visible, but the coating of catalyst particles is thicker, and more of the pore mouths at the filter wall surface are completely filled with catalyst. Finally, Fig. 8 shows a view of the upstream filter wall surface in the heavily coated region 2 . Here, there is a smooth and uniform layer of catalyst particles. None of the underlying substrate structure is visible. A similar appearance is also typical of the downstream filter wall surfaces in region 2 .

\subsection{Mercury Porosimetry}

Figure 9 shows mercury porosimetry data taken for three samples from the three axial regions in one of the square central filter segments. A bi-modal pore size distribution is readily apparent. The larger mode,

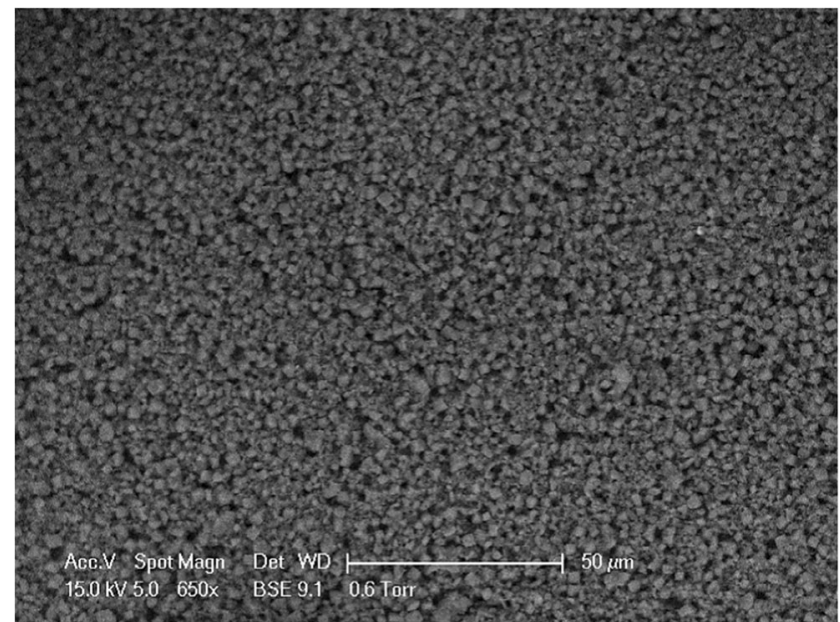

Fig. 8 Electron micrograph of upstream filter wall surface in heavily coated region near inlet end of filter 


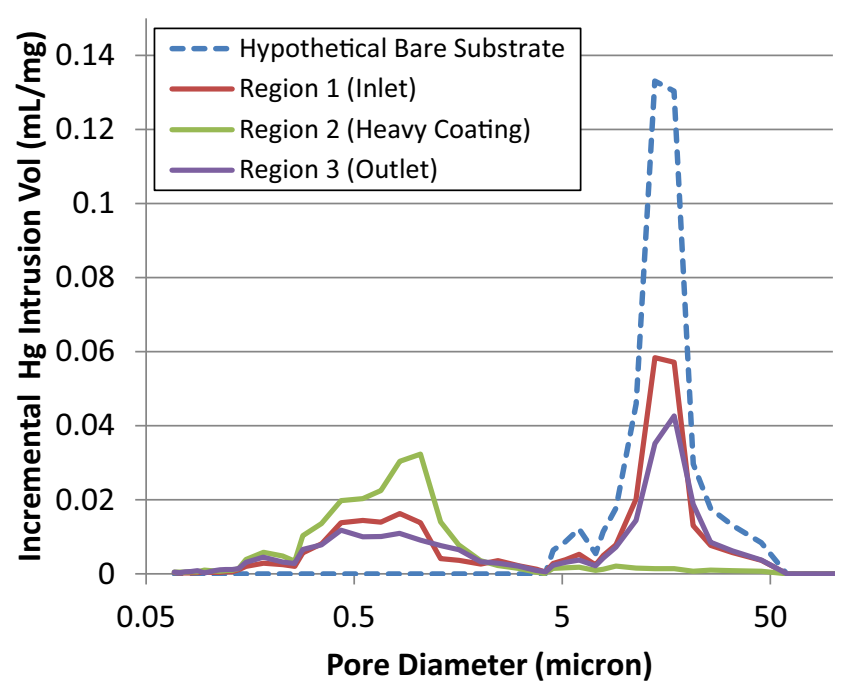

Fig. 9 Pore size distributions in samples from the three axial filter regions

centered at around $15 \mu \mathrm{m}$ on a logarithmic scale, presumably represents the pores in the original $\mathrm{SiC}$ filter substrate. The smaller mode, centered at approximately $0.7 \mu \mathrm{m}$, is likely associated with the catalyst coating. In region 2, there appears to be very little of the original macro-porosity associated with the substrate accessible to the mercury without pushing through pores associated with the catalyst coating. Both modes are apparent in region 1 , with catalyst filling a portion of the pore volume within the filter walls. In region 3, somewhat more of the substrate pore volume has been occupied, assuming that the original porosity of the substrate was the same in all three regions.

The intermediate relationship of region 3 to regions 1 and 2 can be seen more clearly in Fig. 10, which shows the cumulative mercury intrusion volume for the three samples. The dashed lines in both figures represent

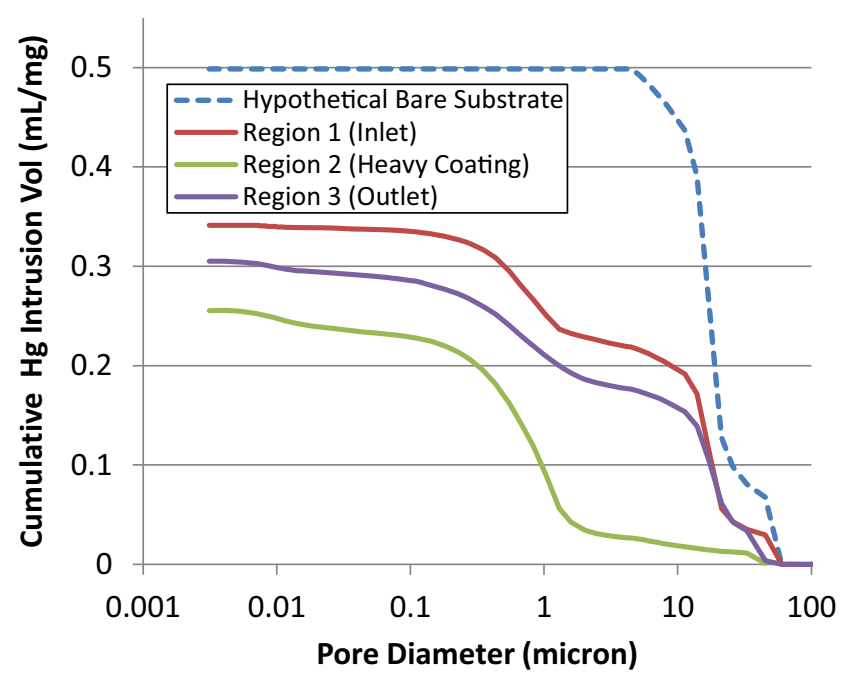

Fig. 10 Cumulative mercury intrusion volume for samples from the three axial filter regions hypothetical pore distributions for the bare substrate, assuming an original porosity of $62 \%$, which would be consistent with advanced high-porosity substrates offered for multi-functional filter applications $[6,10]$.

\subsection{X-ray Tomography}

Figure 11 shows a reconstruction of catalyst location in one of the edge filter segments using X-ray CT data with a resolution of $48.6 \mu \mathrm{m}$. The catalyst is shown in green false color and the filter substrate in blue. Three axial regions similar to those observed visually are apparent. The proportion of bright voxels representing the catalyst coating was averaged along the length of the part from inlet to outlet to generate Fig. 12. As also suggested by relative color in optical images and by proportions of large and small pore volume in the mercury porosimetry data, Fig. 12 indicates relatively light coating in region 1 , heavy coating in region 2 , and intermediate coating in region 3 .

A three-dimensional reconstruction of the transition between region 1 and region 2 is shown in Fig. 13 .

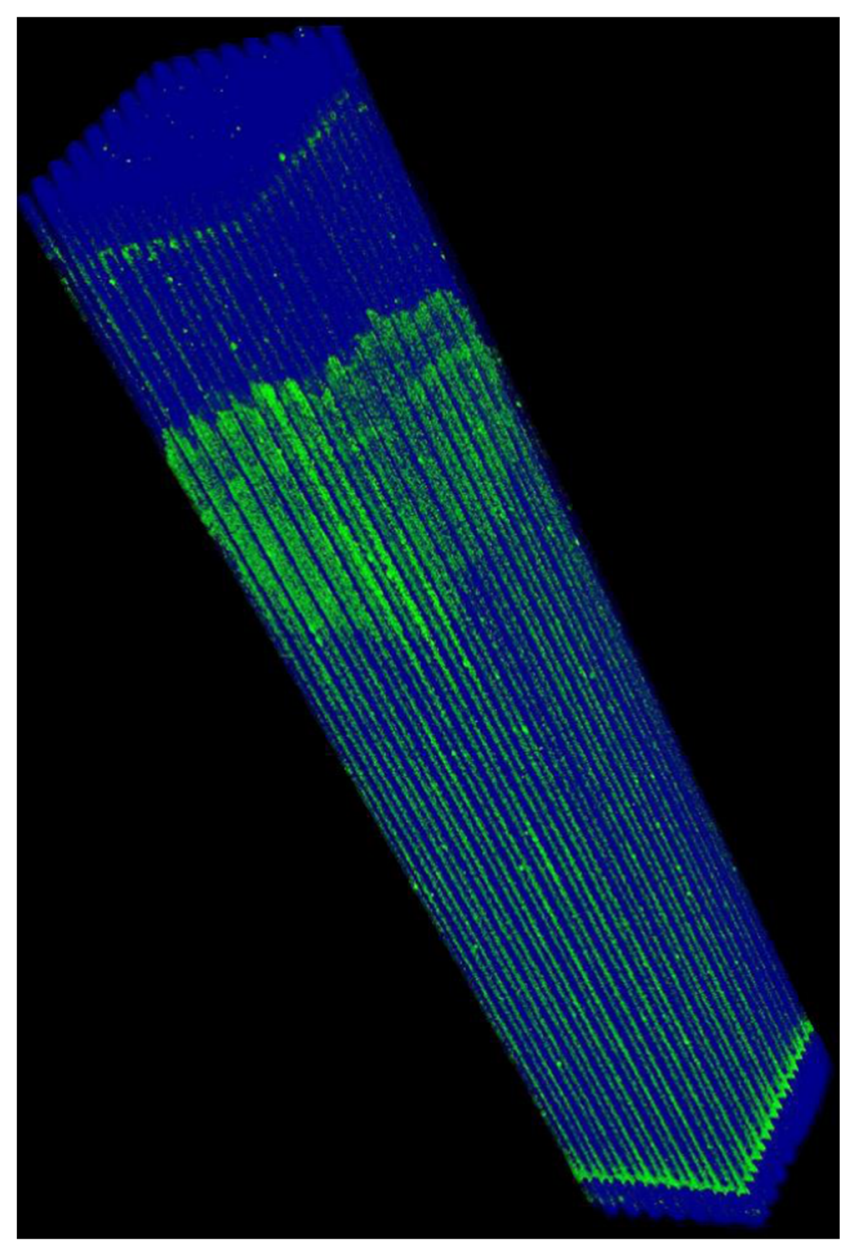

Fig. 11 Low-resolution X-ray CT of one filter segment 


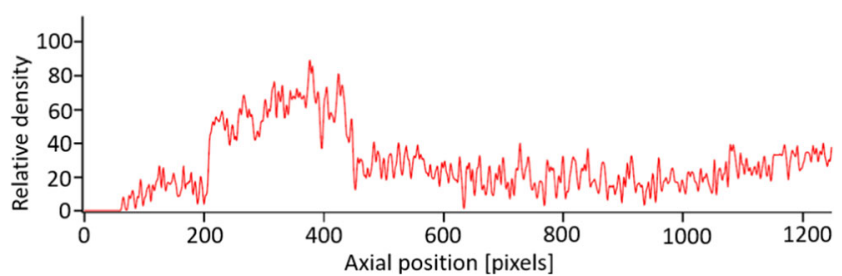

Fig. 12 Axial coating distribution estimated from low-resolution X-ray CT

The catalyst is again shown in green false color, while the filter substrate is shown in blue. This image shows that the surface coating in region 2 is quite thick at some locations, but the coating thickness is not uniform.

Table 1 summarizes mercury porosimetry data used in the analysis of the three two-by-two channel CT datasets. Total porosities ranging from 35 to $42 \%$ reflect the fact that a significant portion of the internal wall porosity is filled with catalyst. The median pore size reported for each region reflects a different weighting between the original macro-porosity in the filter substrate and the much smaller pores within the catalyst deposits. The resolved porosity values shown were found by iterative calculations assuming that voxels associated with the catalyst phase include an unresolved (sub-grid) porosity of $25 \%$. Thus, the resolved porosity values for each dataset are somewhat less than the total porosities measured for samples from the corresponding axial regions.

Figure 14 shows a cross section of the CT data for the two-by-two channel sample taken from region 1 . Catalyst locations are shown in false red color, while original grayscale values are shown for the catalyst substrate. Very little of the catalyst (roughly 5\%) appeared as a coating on the surface of the filter walls in the volume examined. Although most of the catalyst resides within the porous filter wall, the filter walls nevertheless

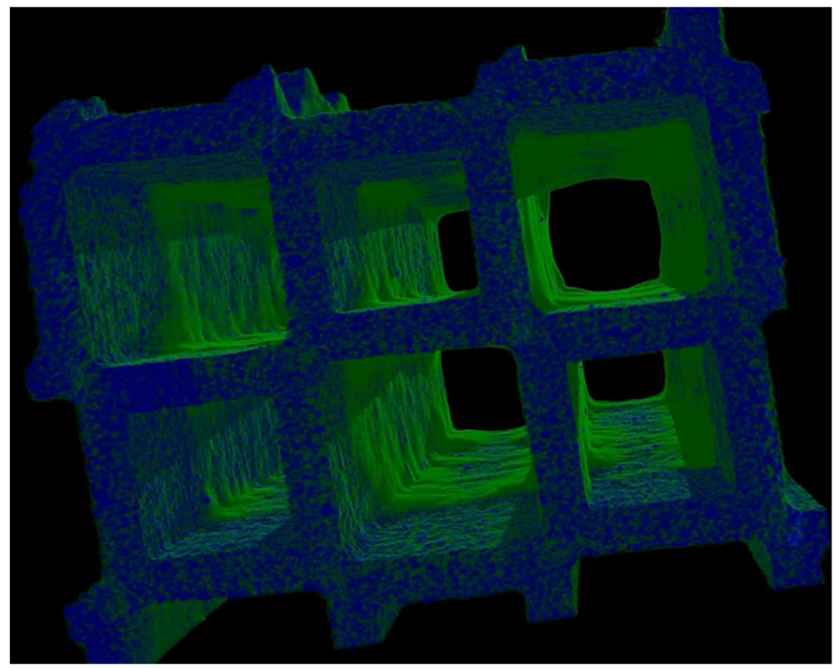

Fig. 13 Reconstruction of transition to heavily coated region
Table 1 Summary of mercury porosimetry and CT data for two-by-two channel sections

\begin{tabular}{llll}
\hline & Region 1 & Region 2 & Region 3 \\
\hline Axial position & Inlet & Near inlet & Middle to outlet \\
Total porosity & $42.4 \%$ & $35.1 \%$ & $37.3 \%$ \\
Median pore size $(\mu \mathrm{m})$ & 14.0 & 0.77 & 11.6 \\
Resolved porosity & $35 \%$ & $26 \%$ & $29 \%$ \\
$\begin{array}{l}\text { Fraction of catalyst } \\
\quad \text { volume within filter walls }\end{array}$ & $95 \%$ & $76 \%$ & $96 \%$ \\
\hline
\end{tabular}

retain significant open porosity. The image also shows variations in the distribution of catalyst within the filter walls. The catalyst seems to radiate outward from the large inlet channels into the wall interiors (as also shown in the optical image included as Fig. 5), suggesting that this region of the filter was coated from the inlet end.

A similar cross section in the heavily coated region 2 is shown as Fig. 15. In contrast with region 1, surface coatings of significant thickness are apparent in this volume on both the upstream and downstream surfaces of the filter walls. Notwithstanding, significant porosity is still apparent within the interior of the filter walls. Macro-porosity is also visible within some of the thick surface coatings. Integrating over the small volume examined indicates that approximately $24 \%$ of the catalyst volume observed resides in coatings on the substrate wall surfaces, rather than within the wall interiors. Coatings seemed to be somewhat thicker in the larger inlet channels.

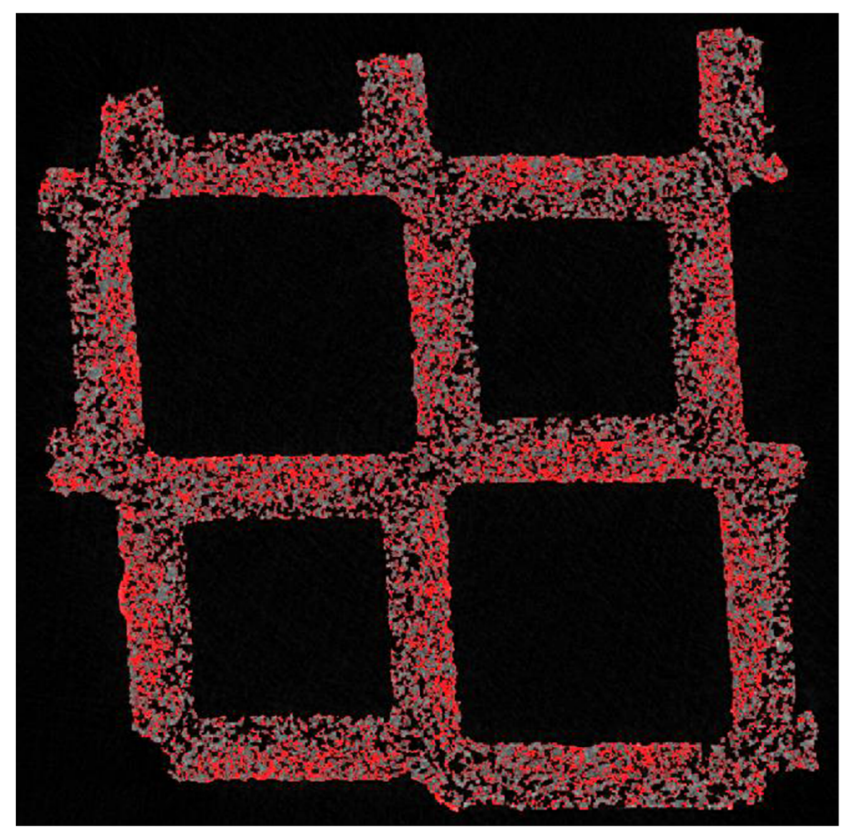

Fig. 14 Cross section of CT data in inlet axial region 


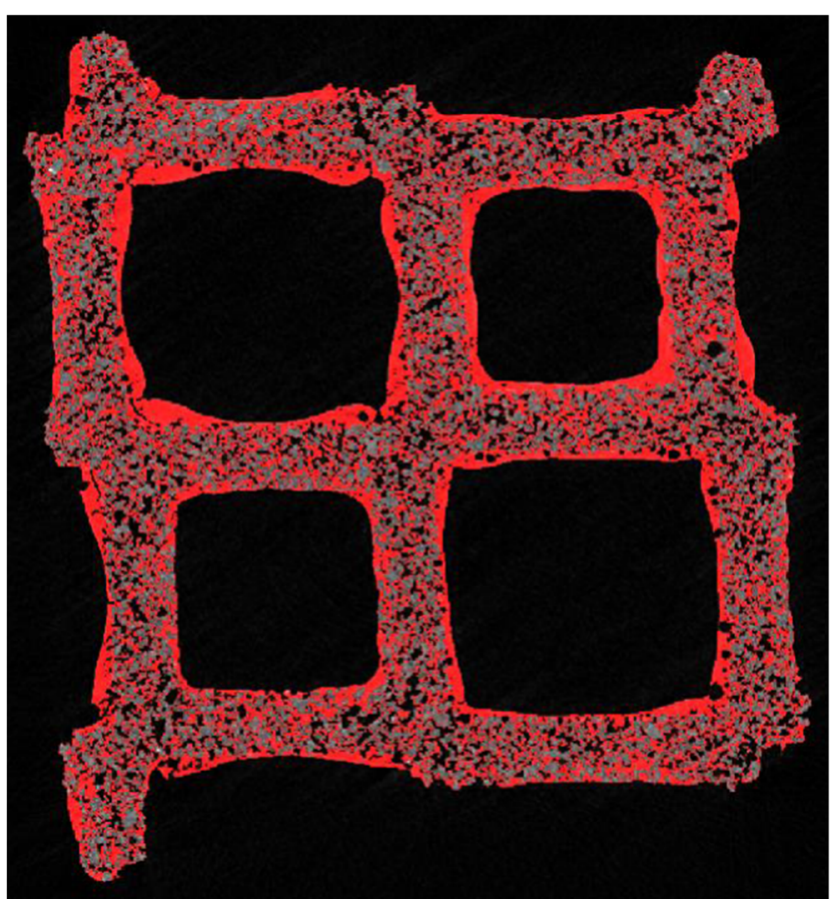

Fig. 15 Cross section of CT data in heavily coated axial region near inlet

Figure 16 shows a cross section from the third and final axial region, which accounts for the majority of the effective length in the device. As in region 1, most of the catalyst volume is within the filter walls, with perhaps only a few percent in thin coatings on the surface. The volume of catalyst present in the wall is somewhat higher. The most striking difference, however, is that the catalyst within the filter wall seems to be

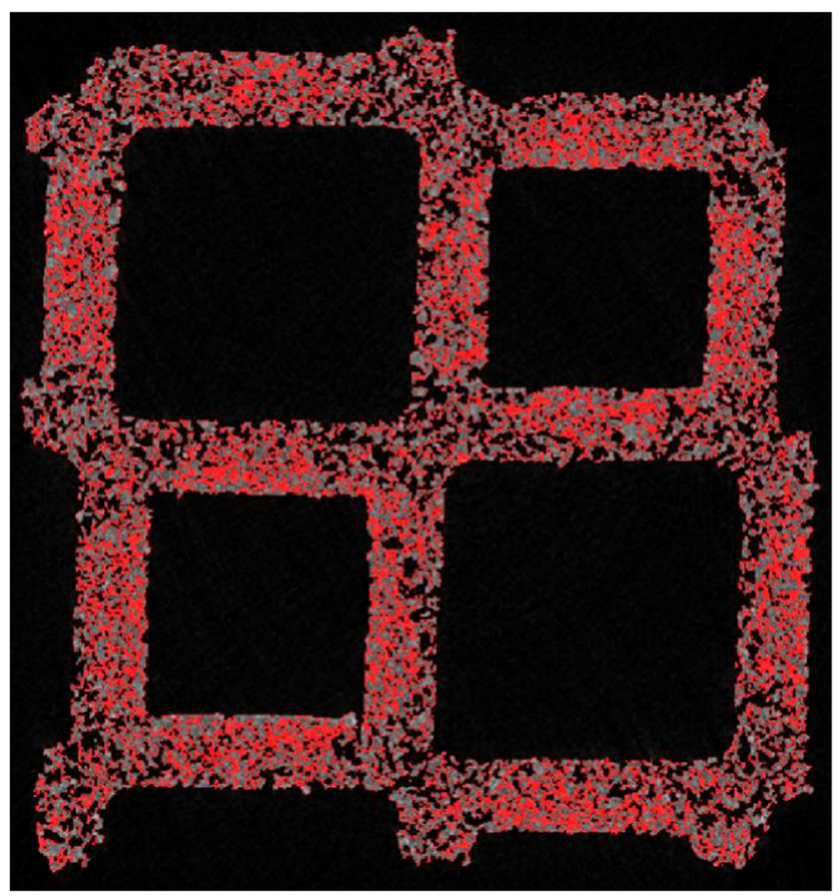

Fig. 16 Cross section of CT data in axial region near outlet

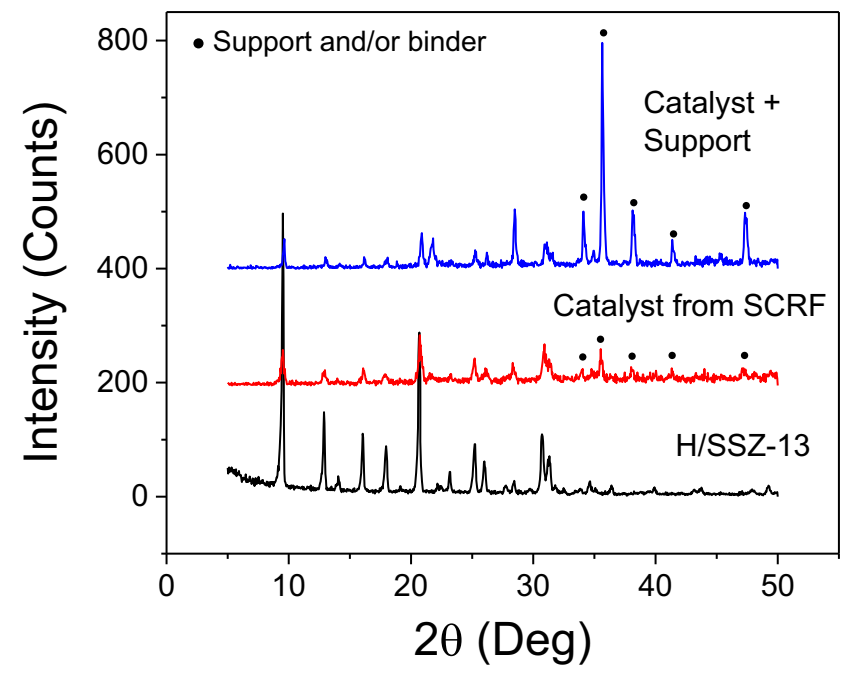

Fig. 17 XRD spectra from SCR filter samples compared to SSZ-13

radiating outward from the smaller outlet channels, indicating coating from the outlet end of the filter brick.

\subsection{Catalyst Composition}

Two samples from the SCR filter were analyzed for information relating to coating composition. One powder sample weighing approximately $30 \mathrm{mg}$ was scraped from both upstream and downstream filter wall surfaces in the heavily coated region 2. A second sample was made up of crushed filter wall material from all three axial regions.

Figure 17 shows XRD patterns for both samples compared to that of a H/SSZ-13 synthesized in-house. Although a number of peaks are attributable to the $\mathrm{SiC}$ support and/or binder material, there are clearly multiple identical diffractions between the in-house SSZ-13 and the two catalyst samples. This indicates that the active SCR phase in the SCR filter adapts an industry standard chabazite substrate, rather than other zeolite supports.

The powder sample scraped from region 2 was also analyzed by X-ray photoelectron spectroscopy. Figure 18

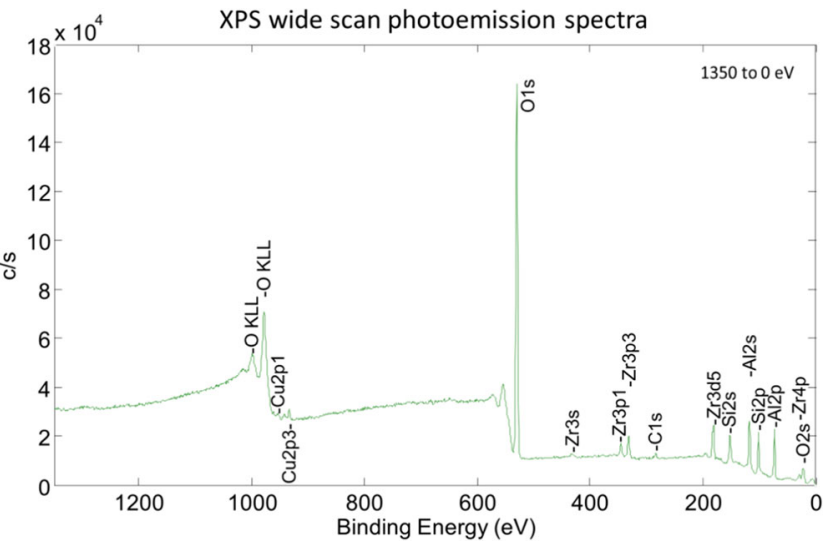

Fig. 18 Wide-scan XPS spectra from catalyst powder sample 
Fig. 19 Narrow-scan XPS spectra from catalyst powder sample
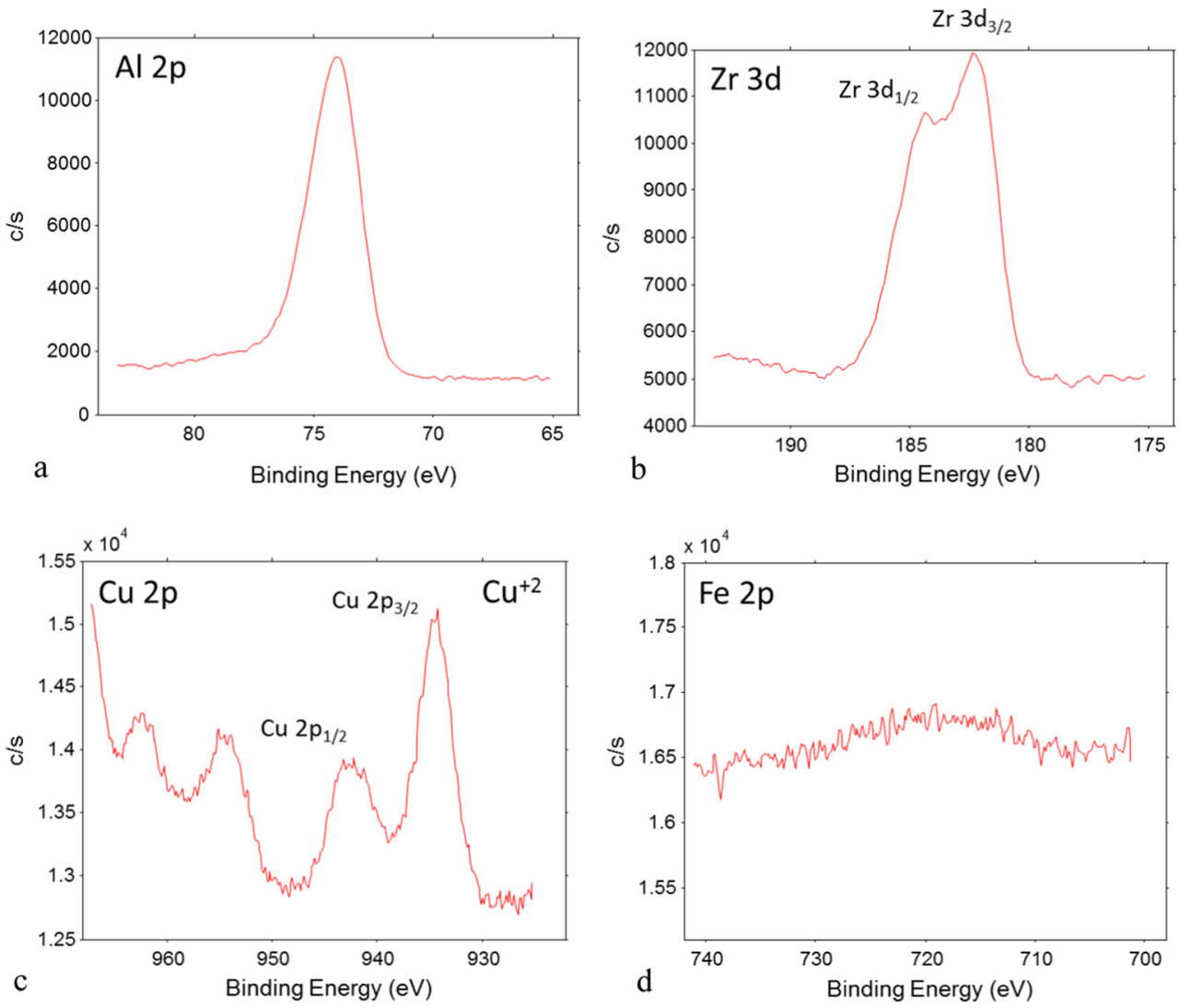

shows a wide-scan XPS spectrum for the sample, and Fig. 19 shows several narrow scan spectra. Table 2 summarizes estimates of near-surface atomic composition for the powder sample. No phosphorus was observed, indicating that the catalyst substrate was SSZ-13, rather than SAPO-34. Copper peaks are readily apparent, but no significant iron was observed, indicating that the active phase in region 2 is entirely $\mathrm{Cu} / \mathrm{SSZ}-13$ rather than $\mathrm{Fe} / \mathrm{SSZ}-13$. The values in Table 2 correspond to a copper weight percentage of approximately 3\%. As expected, there are strong peaks for both silicon and aluminum, but the low $\mathrm{Si} / \mathrm{Al}$ ratio shown in Table 2 suggests that an alumina binder may have been used in addition to the $\mathrm{Cu} / \mathrm{SSZ}$ 13 active phase. Zirconium peaks are also present, which indicates that the catalyst coating also includes some zirconia.

Washcoat distribution suggests that the coating process was carried out in at least two steps, with catalyst slurry being drawn in from both the upstream and downstream ends of the filter, with overlap in the heavily coated region 2 .

Table 2 Near-surface elemental compositions in catalyst powder sample from XPS

\begin{tabular}{llllllll}
\hline Element & $\mathrm{O} \mathrm{1s}$ & $\mathrm{Al} 2 \mathrm{p}$ & $\mathrm{Si} 2 \mathrm{p}$ & $\mathrm{Cu} 1 \mathrm{~s}$ & $\mathrm{Zr} \mathrm{3d}$ & $\mathrm{C} \mathrm{1s}$ & $\mathrm{Fe} 2 \mathrm{p}$ \\
\hline Atomic\% & 67.5 & 17.8 & 10.2 & 0.9 & 1.7 & 1.6 & $<0.3$ \\
\hline
\end{tabular}

Catalyst samples were not obtained exclusively from either region 1 or 3, where virtually all of the catalyst resided within the porous filter walls. However, visual color, $\mathrm{X}$-ray extinction as evidenced in the micro-X-ray $\mathrm{CT}$ images, and washcoat particle size and morphology observed by electron microscopy were all similar between the three regions, suggesting that a similar washcoat composition was used throughout the filter.

\section{Conclusions}

The observations presented here are consistent with three distinct regions in the commercial SCR-coated filter examined, as summarized in Table 3. The first region, located at the inlet of the filter and accounting for approximately 15 to $21 \%$ of the

Table 3 Summary of observed distinct axial regions in coated filter

\begin{tabular}{llll}
\hline & Region 1 & Region 2 & Region 3 \\
\hline $\begin{array}{l}\text { Axial position } \\
\begin{array}{c}\text { Approximate } \\
\text { fraction of total } \\
\text { effective axial length }\end{array}\end{array}$ & Inlet & Near inlet & Middle to outlet \\
$\begin{array}{c}\text { Likely direction } \\
\text { of coating }\end{array}$ & From inlet & From both inlet & From outlet \\
$\begin{array}{c}\text { Relative catalyst } \\
\text { loading }\end{array}$ & Light & $\begin{array}{c}\text { and outlet } \\
\text { Heavy }\end{array}$ & Intermediate \\
\hline
\end{tabular}


total effective filter length, is relatively lightly coated. The catalyst is present mostly within the filter walls, with higher concentrations near the upstream wall surfaces.

The second region, accounting for 14 to $20 \%$ of the total effective filter length, has a relatively heavy coating of catalyst. Some larger pores are apparent in the interior of the filter wall in X-ray CT images (a few are even visible within thick catalyst deposits), but relatively little of this volume was apparent as large pores in the mercury porosimetry data, presumably because it was effectively screened by smaller pores in the thick catalyst coatings on both sides of the filter walls and by internal deposits closer to the surfaces of the sample.

The third region, accounting for the remaining effective length (approximately $65-70 \%$ of the total), has an intermediate loading relative to the first two regions, with a gradient from the downstream surfaces of the filter walls to the upstream surfaces.

The observed distribution of catalyst within the device is consistent with a multi-stage coating process from both ends of the filter. One or more coating operations from the inlet end would place catalyst on the upstream sides of the filter walls in regions 1 and 2, and one or more coating operations from the outlet end would place catalyst on the downstream sides of filter walls in regions 3 and 2 .

Chemical analysis by XRD and XPS indicates that the active phase in the filter coating is $\mathrm{Cu} / \mathrm{SSZ}-13$. Iron concentration was insignificant. Alumina may have been used as a binder in the coating, and some zirconia is also evident.

The present study does not extend to the effects of the observed distribution of catalyst within the device. Given the relative scarcity of accessible large pores in region 2, it is likely that the permeability is considerably lower than in the other two regions. There may also be a difference in permeability between the somewhat more lightly loaded region 1 and the more heavily loaded region 3. Other studies [5] have shown that the direction of a catalyst concentration gradient across the thickness of a filter wall can have a significant impact on pressure drop as soot is deposited. Thus, both initial permeability and orientation of the coating across the filter walls in the three regions could affect distribution of soot in the device and overall pressure drop during operation.

Modeling studies in the literature [8] have also suggested that differences in catalyst loading along the axial length of a multi-functional filter can affect reactant concentrations moving through the device, and to some extent, overall conversion under some operating conditions.

However, it should be noted that the third region accounted for the majority of the effective device length (65-70\%). In a simple model of this particular device, parametric values taken from this region would be most representative. The orientation of the catalyst coating in this region (heavier on the downstream side) was shown to result in lower-pressure drop during soot loading in Rappé (2014) [5]. Further experimental and modeling studies would be needed to determine whether any effects of the observed coating distribution in this product have a significant impact on performance.

In any case, as more advanced catalytic aftertreatment devices are developed and deployed, it is likely that ceramic substrate manufacturers and catalyst coaters will benefit from accurate and comprehensive characterization of catalyst distribution at length scales down to those of individual pores and catalyst particles. This information could also be valuable for vehicle manufacturers as they seek to better understand component design features and system behavior. A battery of techniques such as those presented here, including visual examination of sectioned samples, mercury porosimetry, microscopy, and micro-X-ray CT, can help provide such insight.

Acknowledgements A portion of this research was performed using EMSL, a DOE Office of Science User Facility sponsored by the Office of Biological and Environmental Research. A portion of the X-ray CT imaging for this study was performed by Brandon Walters at Micro Photonics. Thanks to Ken Howden and Gurpreet Singh at the US Department of Energy Office of Vehicle Technologies for funding the CLEERS program. Thanks to Dana Ruane of PNNL for assistance in obtaining the VW aftertreatment module. Special thanks to Dr. Christine Lambert of Ford and Dr. Timothy Johnson, Dr. Ameya Joshi, and Dr. Todd St. Clair of Corning for helpful discussions on coated filter microstructure.

\section{Compliance with Ethical Standards}

Conflict of Interest The authors declare that they have no competing interests.

Open Access This article is distributed under the terms of the Creative Commons Attribution 4.0 International License (http:// creativecommons.org/licenses/by/4.0/), which permits unrestricted use, distribution, and reproduction in any medium, provided you give appropriate credit to the original author(s) and the source, provide a link to the Creative Commons license, and indicate if changes were made.

\section{References}

1. Richter, J.M., et al.: Application of Catalyzed Gasoline Particulate Filters to GDI Vehicles. SAE Technical Paper, 2012-01-1244 (2012)

2. Lee, J.H., Paratore, M. and Brown, D.: Evaluation of Cu-based SCR/DPF Technology for Diesel Exhaust Emission Control. SAE Technical Paper, 2008-01-0072 (2008)

3. Lambert, C.K., et al.: Analysis of Ash in Low Mileage, Rapid Aged, and High Mileage Gasoline Exhaust Particle Filters. SAE Int. J. Engines. 10(4) (2017)

4. Cavataio, G., Girard J.W., and Lambert, C.K.: Cu/Zeolite SCR on High Porosity Particulate Filters: Laboratory and Engine Performance Evaluations. SAE Technical Paper, 2009-01-0897 (2009)

5. Rappé, K.G.: Integrated selective catalytic reduction-diesel particulate filter Aftertreatment: insights into pressure drop, $\mathrm{NO}_{\mathrm{x}}$ conversion, and passive soot oxidation behavior. Ind. Eng. Chem. Res. 53(45), 17547-17557 (2014) 
6. Cumaranatunge, L., Chiffey, A., Stetina, J., McGonigle, K., Repley, G., Lee, A., Chatterjee, S.: A study of the soot combustion efficiency of an $\mathrm{SCRF}^{\circledR}$ catalyst vs a CSF during active regeneration. Emiss. Control Sci. Technol. 3(1), 93-104 (2017)

7. Mihai, O., et al.: The effect of soot on ammonium nitrate species and $\mathrm{NO} 2$ selective catalytic reduction over cu-zeolite catalyst-coated particulate filter. Phil. Trans. R. Soc. A, 374(2061), 14 (2016)

8. Karamitros, D., Koltsakis, G.: Model-based optimization of catalyst zoning on SCR-coated particulate filters. Chem. Eng. Sci. 173, 514-524 (2017)
9. Kamp, C., et al., Ash Permeability Determination in the Diesel Particulate Filter from Ultra-high Resolution 3D X-Ray Imaging and Image-Based Direct Numerical Simulations. SAE Int. J. Fuels Lubr., .10(2) (2017). https://doi.org/10.4271/2017-01-0927

10. Mey, D., et al., Improved DPF Substrate for Washcoat Accomodation. SAE Technical Paper. 2009-01-0288 (2009). https://doi.org/10.4271/2009-01-0288

11. Dudák, M., et al.: Impact of zeolite and $\gamma$-alumina intra-particle diffusion on the performance of a dual layer catalyst. Chem. Eng. J. 301(Supplement C), 178-187 (2016) 